\title{
Updated Neuronal Scaling Rules for the Brains of Glires (Rodents/Lagomorphs)
}

\author{
Suzana Herculano-Houzel ${ }^{a, b}$ Pedro Ribeiro ${ }^{a, b}$ Leandro Campos ${ }^{a, b}$ \\ Alexandre Valotta da Silva ${ }^{b, c}$ Laila B. Torres ${ }^{b, c}$ Kenneth C. Cataniad Jon H. Kaas ${ }^{e}$ \\ a Instituto de Ciências Biomédicas, Universidade Federal do Rio de Janeiro and ${ }^{\mathrm{b}}$ Instituto Nacional de Neurociência \\ Translacional, Rio de Janeiro, and ' Departamento de Biociências, Universidade Federal de São Paulo - Campus

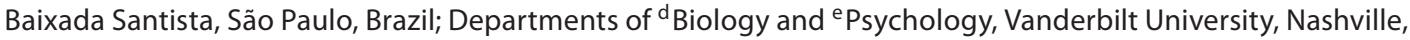 \\ Tenn., USA
}

\section{Key Words}

Rodents $\cdot$ Brain size $\cdot$ Evolution $\cdot$ Neurons $\cdot$ Glia $\cdot$ Glires

\begin{abstract}
Brain size scales as different functions of its number of neurons across mammalian orders such as rodents, primates, and insectivores. In rodents, we have previously shown that, across a sample of 6 species, from mouse to capybara, the cerebral cortex, cerebellum and the remaining brain structures increase in size faster than they gain neurons, with an accompanying decrease in neuronal density in these structures [Herculano-Houzel et al.: Proc Natl Acad Sci USA 2006;103:12138-12143]. Important remaining questions are whether such neuronal scaling rules within an order apply equally to all pertaining species, and whether they extend to closely related taxa. Here, we examine whether 4 other species of Rodentia, as well as the closely related rabbit (Lagomorpha), conform to the scaling rules identified previously for rodents. We report the updated neuronal scaling rules obtained for the average values of each species in a way that is directly comparable to the scaling rules that apply to primates [Gabi et al.: Brain Behav Evol 2010;76:32-44], and examine whether the scaling relationships are affected when phylogenetic relatedness in the dataset is accounted for. We have found that the brains of the spiny rat, squirrel, prairie
\end{abstract}

dog and rabbit conform to the neuronal scaling rules that apply to the previous sample of rodents. The conformity to the previous rules of the new set of species, which includes the rabbit, suggests that the cellular scaling rules we have identified apply to rodents in general, and probably to Glires as a whole (rodents/lagomorphs), with one notable exception: the naked mole-rat brain is apparently an outlier, with only about half of the neurons expected from its brain size in its cerebral cortex and cerebellum.

Copyright $\odot 2011$ S. Karger AG, Basel

\section{Introduction}

Rodents are a monophyletic group [Lin et al., 2002] which originated about 65 million years ago, in Laurasia [Douzery et al., 2003; Horner et al., 2007], and which now occupy a number of different niches on all continents. At over 2,200 species, rodents make up the largest order of mammals, representing over $40 \%$ of all mammalian species [Wilson and Reeder, 2005], and vary in adult size from about $7 \mathrm{~g}$ (African pigmy mouse) to over $70 \mathrm{~kg}$ (capybara). What are the rules and constraints that apply to how their bodies and brains are built, and how are they different from or similar to the rules that apply to other mammals?

\section{KARGER}

() 2011 S. Karger AG, Basel

Fax +4161306 1234

E-Mail karger@karger.ch

www.karger.com
Accessible online at: www.karger.com/bbe
Suzana Herculano-Houzel

Instituto de Ciências Biomédicas, UFRJ

Av. Carlos Chagas Filho, 373

Ilha do Fundão, Rio de Janeiro, RJ 21941-902 (Brazil)

Tel. +55 212562 6390, E-Mail suzanahh@ gmail.com 
It is often considered that mammalian brains in general vary in size as a power function of body size [Martin, 1981; Fox and Wilczynski, 1986], and that larger brains are composed of relatively larger cerebral cortices, a cerebellum of constant relative size [Stephan et al., 1981; Clark et al., 2001], larger neurons, resulting in lesser neuronal densities, and increasing glia/neuron ratios [Tower and Elliot, 1952; Haug, 1987; Stolzenburg et al., 1989; Marino, 2006].

Applying a novel method developed by our group that allows for the fast and reliable estimation of numbers of neuronal and nonneuronal cells in any dissectable structure, the isotropic fractionator [Herculano-Houzel and Lent, 2005], we have been able to determine the cellular scaling rules that apply to rodent brains. Briefly, rodent cerebral cortex and cerebellum increase in size as a function of their numbers of neurons raised to large powers (1.8 and 1.4, respectively), while neuronal densities decrease and average neuronal sizes and the nonneuronal/ neuronal cell ratios increase [Herculano-Houzel et al., 2006]. Rodents, therefore, seem to conform to the general rules obtained from a combination of mammals from several orders [Haug, 1987]. In contrast, we later found that primate cerebral cortex and cerebellum increase in size as linear functions of their numbers of neurons, with insignificant changes in neuronal density, average neuronal size, and nonneuronal/neuronal cell ratios [Herculano-Houzel et al., 2007; Gabi et al., 2010]. In Eulipotyphla (insectivores), on the other hand, the cerebellum increases linearly in mass with its number of neurons, as in primates, while the mass of the cerebral cortex scales hypermetrically with its number of neurons, as in rodents [Sarko et al., 2009].

While the cellular scaling rules for cerebral cortex and cerebellum differ across the three orders, recent evidence raised the possibility that the scaling rules for the remaining areas might be shared across them. In rodents, we reported originally that the ensemble of brainstem, diencephalon, basal ganglia and olfactory bulb (called 'rest of brain' or RoB) scales in mass as a function of its number of neurons raised to a large exponent of approximately 1.8 [Herculano-Houzel et al., 2006]. In primates, our first study suggested that the mass of the RoB varied linearly with its number of neurons [Herculano-Houzel et al., 2007]. However, we recently found that, with a larger sample size of 11 species that included humans, and after correcting for phylogenetic relatedness in the dataset, the variations in the mass of the primate RoB can be described as a power function of its number of neurons with an exponent of 1.4 [Gabi et al., 2010], more similar to rodents, although they are still best fit as a linear function of the number of neurons in the structure. In Eulipotyphla, as in primates, we also found that variations in the mass of RoB are best described as a linear function of its number of neurons [Sarko et al., 2009]. However, the data points for the RoB are much more scattered than for the other brain structures, and there is substantial overlap among the three orders that suggests that the neuronal scaling rules for this part of the brain might actually be shared across rodents, primates and insectivores.

A proper, direct comparison across the scaling rules that apply to these three mammalian orders is, however, currently hampered by differences in the way the scaling rules were initially reported for rodents. In our initial study, we reported the power laws that describe variations in the mass of different rodent brain structures as a function of their numbers of cells derived from individual values rather than species averages, and included the olfactory bulb in the RoB [Herculano-Houzel et al., 2006]. In subsequent studies, we have calculated the power laws that apply to species averages, not individuals, which eliminates the confounding factor of intraspecific scaling, and systematically excluded the olfactory bulb from the RoB, given that the olfactory bulb is often not available for analysis [Herculano-Houzel et al., 2007; Azevedo et al., 2009; Sarko et al., 2009; Gabi et al., 2010]. It is necessary, therefore, to examine the cellular scaling laws that apply to rodents in a manner that can be directly comparable to present and future datasets on other mammalian groups.

Given the large diversity of rodent species and the niches they occupy, other interesting issues are whether the cellular scaling rules that we identified for the initial 6 rodent species also apply to rodents in general, whether they are affected once phylogenetic relationships are taken into consideration, and whether they extend to a related clade, the lagomorphs, which together with rodents compose the monophyletic group Glires [Liu and Miyamoto, 1999; Douzery and Huchon, 2004; Kullberg et al., 2006].

Here, we update the scaling laws that apply to the previous dataset of 6 rodent species by using species averages and excluding the olfactory bulb from the RoB. This yields scaling laws that can be directly compared with our data from primates [Gabi et al., 2010] and insectivores [Sarko et al., 2009]. Next, we extended our previous study by applying the same method, the isotropic fractionator, to estimate total numbers of neuronal and nonneuronal cells in the cerebral cortex, cerebellum and the RoB, and separately in the olfactory bulb, and to determine how 
Table 1. Average data for the 11 species of Glires

\begin{tabular}{lccccc}
\hline & Naked mole-rat & Mouse & Hamster & Rat & Spiny rat \\
\hline Number & 3 & 4 & 2 & 4 & 2 \\
\hline Body mass, g & $23.3 \pm 5.9$ & $40.4 \pm 11.6$ & $168.1 \pm 13.6$ & $315.1 \pm 102.9$ & $223.5 \pm 16.6$ \\
Brain mass, g & $0.392 \pm 0.045$ & $0.402 \pm 0.028$ & $0.965 \pm 0.136$ & $1.724 \pm 0.292$ & $2.078 \pm 0.071$ \\
Brain neurons & $26.88 \pm 3.34 \times 10^{6}$ & $67.87 \pm 10.41 \times 10^{6}$ & $84.22 \pm 9.89 \times 10^{6}$ & $188.87 \pm 12.62 \times 10^{6}$ & $202.09 \pm 2.93 \times 10^{6}$ \\
Brain other cells & $24.19 \pm 1.74 \times 10^{6}$ & $33.86 \pm 6.66 \times 10^{6}$ & $70.64 \pm 11.94 \times 10^{6}$ & $121.91 \pm 7.11 \times 10^{6}$ & $181.27 \pm 4.31 \times 10^{6}$ \\
\hline Cortical mass, g & $0.184 \pm 0.026$ & $0.173 \pm 0.015$ & $0.446 \pm 0.048$ & $0.769 \pm 0.113$ & $0.924 \pm 0.050$ \\
Cortical neurons & $6.15 \pm 1.06 \times 10^{6}$ & $13.69 \pm 2.24 \times 10^{6}$ & $17.14 \pm 3.62 \times 10^{6}$ & $31.02 \pm 3.03 \times 10^{6}$ & $26.09 \pm 2.16 \times 10^{6}$ \\
Cortical other cells & $8.40 \pm 1.20 \times 10^{6}$ & $12.06 \pm 3.67 \times 10^{6}$ & $41.87 \pm 1.35 \times 10^{6}$ & $45.69 \pm 5.68 \times 10^{6}$ & $71.83 \pm 6.71 \times 10^{6}$ \\
\hline Cerebellar mass, g & $0.048 \pm 0.004$ & $0.056 \pm 0.005$ & $0.145 \pm 0.030$ & $0.272 \pm 0.038$ & $0.330 \pm 0.026$ \\
Cerebellar neurons & $15.74 \pm 2.85 \times 10^{6}$ & $42.22 \pm 9.28 \times 10^{6}$ & $61.21 \pm 12.35 \times 10^{6}$ & $139.17 \pm 11.18 \times 10^{6}$ & $162.51 \pm 3.55 \times 10^{6}$ \\
Cerebellar other cells & $5.48 \pm 1.27 \times 10^{6}$ & $6.95 \pm 1.50 \times 10^{6}$ & $7.43 \pm 1.71 \times 10^{6}$ & $29.00 \pm 6.28 \times 10^{6}$ & $36.37 \pm 5.09 \times 10^{6}$ \\
\hline RoB mass, g & $0.160 \pm 0.019$ & $0.172 \pm 0.019$ & $0.375 \pm 0.057$ & $0.683 \pm 0.153$ & $0.824 \pm 0.006$ \\
RoB neurons & $4.98 \pm 0.85 \times 10^{6}$ & $11.96 \pm 1.58 \times 10^{6}$ & $5.87 \pm 1.16 \times 10^{6}$ & $18.68 \pm 4.24 \times 10^{6}$ & $13.49 \pm 1.53 \times 10^{6}$ \\
RoB other cells & $10.31 \pm 1.84 \times 10^{6}$ & $14.85 \pm 3.59 \times 10^{6}$ & $21.33 \pm 8.88 \times 10^{6}$ & $47.22 \pm 5.98 \times 10^{6}$ & $73.07 \pm 2.69 \times 10^{6}$ \\
\hline Olf bulb mass, g & $0.021 \pm 0.001$ & $0.014 \pm 0.004$ & $0.055 \pm 0.011$ & $0.077 \pm 0.023$ \\
Olf bulb neurons & $2.30 \pm 0.64 \times 10^{6}$ & $3.89 \pm 1.25 \times 10^{6}$ & $5.75 \pm 0.35 \times 10^{6}$ & $11.26 \pm 3.68 \times 10^{6}$ & 0.132 \\
Olf bulb other cells & $3.57 \pm 1.55 \times 10^{6}$ & $5.46 \pm 1.15 \times 10^{6}$ & $5.51 \pm 2.28 \times 10^{6}$ & $9.60 \pm 2.42 \times 10^{6}$ & $21.13 \times 10^{6}$ \\
\hline
\end{tabular}

All data are averages \pm standard deviation. Averages for mouse, hamster, rat, guinea pig, agouti and capybara were calculated from Herculano-Houzel et al. [2006]. RoB and whole brain do not include the olfactory bulb. n.a. = Not available; Olf $=$ olfactory.

these numbers scale together with brain size in 5 other species: the Amazonian ground-dwelling spiny rat (Proechimys cayennensis), the North American prairie dog (Cynomys sp.), the North American grey squirrel (Sciurus carolinensis) and the African naked mole-rat (Heterocephalus glaber), as well as the domestic rabbit (Oryctolagus cuniculus), a lagomorph. We then determine whether the brains of these 5 species conform to the rodent scaling rules, apply the analysis of independent contrasts to control for phylogenetic relatedness in the expanded dataset, and provide an updated set of scaling rules for Glires, to be compared to the rules that apply to Primata and Eulipotyphla.

\section{Materials and Methods}

\section{Animals}

Three adult male naked mouse-rats (H. glaber), 2 adult male spiny rats (Proechimys cayennensis), 3 North American prairie dogs (Cynomys sp., 2 males and 1 female), 3 North American grey squirrels (S. carolinensis, sex unknown) and 1 adult male laboratory rabbit (O. cuniculus) were analyzed. At this point, eventual systematic differences between males and females in the cellular composition of their brains can neither be examined nor ruled out, given the predominance of males in our sample. However, we expect that, due to the small intraspecific variability observed in brain mass and number of neurons (of typically less than 15\%; see table 1), any eventual such sex differences will be probably relatively small compared to the variations of nearly 4 orders of magnitude analyzed here, and therefore of little impact towards our conclusions. Naked mole-rats ( 3 males of 4-8 months of age) were obtained from colonies in the Department of Biology at Vanderbilt University; spiny rats were obtained from colonies in the Federal University of São Paulo; prairie dogs were kindly provided by Dr. Mohammad Abedin at Drexel University College of Medicine, Philadelphia, Pa., USA; grey squirrels were wild-caught in Nashville, Tenn., USA, and the rabbit was obtained from the colony of the Institute of Medical Biochemistry at the Federal University of Rio de Janeiro. All animals bred in captivity were young adults of a few months of age; the age of wild-caught animals could not be determined. While the joint analysis of wild-caught and captivity-bred animals is not ideal, because of possible changes in brain and body size associated with domestication, the reduction of body size-corrected brain mass in Glires has been reported to be very small or even negligible, i.e. $0 \%$ in the mouse, $8 \%$ in the laboratory rat, and $13 \%$ in the lab rabbit [Kruska, 2007]. Because this variation is in the same range of intraspecific variation found in our sample (table 1) and much smaller than the over 1,000-fold variation across species, we believe that the comparison of captive and wild-caught species is therefore warranted.

Both hemispheres were available for 1 spiny rat and 1 squirrel, and the numbers provided refer to the sum of both hemispheres; for all other animals, data are expressed as twice the numbers obtained for the available hemispheres. This is warranted by our 


\begin{tabular}{lllllc}
\hline Guinea pig & Prairie dog & Grey squirrel & Rabbit & Agouti & Capybara \\
\hline 2 & 3 & 3 & 1 & 3 & 2 \\
\hline $311.0 \pm 49.1$ & $1,515.0 \pm 230.6$ & ca. 500 & 4,600 & $2,843.3 \pm 195.5$ & $47,500.0 \pm 3,535.5$ \\
$3.656 \pm 0.486$ & $5.321 \pm 0.197$ & $5.548 \pm 0.306$ & 9.132 & $17.628 \pm 1.900$ & $74.734 \pm 3.756$ \\
$233.56 \pm 4.08 \times 10^{6}$ & $473.94 \pm 78.74 \times 10^{6}$ & $453.66 \pm 59.75 \times 10^{6}$ & $494.20 \times 10^{6}$ & $795.11 \times 10^{6}$ & $1,572.56 \pm 72.64 \times 10^{6}$ \\
$228.09 \pm 9.14 \times 10^{6}$ & $417.35 \pm 27.35 \times 10^{6}$ & $530.48 \pm 5.60 \times 10^{6}$ & $630.80 \times 10^{6}$ & $951.68 \times 10^{6}$ & $3,197.93 \pm 974.58 \times 10^{6}$ \\
\hline $1.938 \pm 0.231$ & $2.586 \pm 0.109$ & $2.730 \pm 0.178$ & 4.448 & $8.913 \pm 1.214$ & $48.175 \pm 2.714$ \\
$43.51 \pm 3.17 \times 10^{6}$ & $53.77 \pm 6.04 \times 10^{6}$ & $77.33 \pm 2.63 \times 10^{6}$ & $71.45 \times 10^{6}$ & $110.64 \pm 2.58 \times 10^{6}$ & $306.50 \pm 62.73 \times 10^{6}$ \\
$108.61 \pm 12.78 \times 10^{6}$ & $183.45 \pm 17.96 \times 10^{6}$ & $209.65 \pm 13.00 \times 10^{6}$ & $254.80 \times 10^{6}$ & $416.21 \pm 0.95 \times 10^{6}$ & $1,847.82 \pm 512.39 \times 10^{6}$ \\
\hline $0.500 \pm 0.077$ & $0.789 \pm 0.093$ & $0.874 \pm 0.069$ & 1.412 & $2.742 \pm 0.196$ & $6.632 \pm 1.312$ \\
$167.85 \pm 2.18 \times 10^{6}$ & $350.08 \pm 72.18 \times 10^{6}$ & $342.83 \pm 71.18 \times 10^{6}$ & $396.67 \times 10^{6}$ & $673.49 \pm 48.14 \times 10^{6}$ & $1,157.81 \pm 5.52 \times 10^{6}$ \\
$36.29 \pm 4.51 \times 10^{6}$ & $66.16 \pm 39.27 \times 10^{6}$ & $110.80 \pm 9.07 \times 10^{6}$ & $124.58 \times 10^{6}$ & $155.99 \pm 32.98 \times 10^{6}$ & $570.94 \pm 81.10 \times 10^{6}$ \\
\hline $1.218 \pm 0.178$ & $1.945 \pm 0.146$ & $1.943 \pm 0.146$ & 3.272 & $5.972 \pm 0.514$ & $19.927 \pm 0.270$ \\
$22.19 \pm 9.43 \times 10^{6}$ & $34.09 \pm 0.59 \times 10^{6}$ & $33.49 \pm 17.13 \times 10^{6}$ & $26.08 \times 10^{6}$ & $43.20 \times 10^{6}$ & $108.25 \pm 4.40 \times 10^{6}$ \\
$83.18 \pm 8.14 \times 10^{6}$ & $167.74 \pm 5.02 \times 10^{6}$ & $210.02 \pm 3.42 \times 10^{6}$ & $251.42 \times 10^{6}$ & $356.84 \times 10^{6}$ & $779.17 \pm 381.09 \times 10^{6}$ \\
\hline $0.103 \pm 0.013$ & n.a. & $0.226 \pm 0.019$ & 0.156 & $0.737 \pm 0.162$ & $1.302 \pm 0.031$ \\
$6.06 \pm 1.30 \times 10^{6}$ & n.a. & $25.10 \pm 9.14 \times 10^{6}$ & $18.76 \times 10^{6}$ & $58.12 \pm 4.95 \times 10^{6}$ & $28.56 \pm 8.52 \times 10^{6}$ \\
$10.15 \pm 4.22 \times 10^{6}$ & n.a. & $39.92 \pm 15.87 \times 10^{6}$ & $22.94 \times 10^{6}$ & $72.60 \pm 19.68 \times 10^{6}$ & $67.39 \pm 25.02 \times 10^{6}$ \\
\hline
\end{tabular}

finding that there are no systematic differences between total numbers of neurons between the two hemispheres at least in mice and rats [Herculano-Houzel et al., unpubl. observations]. Additionally, we analyzed the olfactory bulbs of 2 other grey squirrels and 2 adult male Swiss mice (Mus musculus, obtained from the colony at the Institute of Biomedical Sciences, UFRJ). Body weights for Sciurus were not recorded, and therefore an average of $500 \mathrm{~g}$ was considered. All veterinary care and procedures reported herein were performed according to the ethical standards of the Committee for the Ethical Use of Research Animals (CEUP) of the Federal University of Rio de Janeiro.

Here, we also examine data reported previously by our own group for 4 Swiss mice (M. musculus), 2 golden hamsters (Mesocricetus auratus), 4 Wistar rats (Rattus norvegicus), 2 guinea pigs (Cavia porcellus), 3 agoutis (Dasyprocta primnolopha) and 2 capybaras (Hydrochoerus hydrochaeris [Herculano-Houzel et al., 2006]). In the original report, RoB corresponded to the sum of the olfactory bulb (which had been analyzed separately for all individuals except for mice) and the ensemble of brainstem, diencephalon, and basal ganglia. Here, we report the values for the olfactory bulbs separately from the RoB, which, as described in primates and insectivores [Herculano-Houzel et al., 2007; Sarko et al., 2009; Gabi et al., 2010], amounts to the ensemble of brainstem, diencephalon, and basal ganglia. For the mouse, we subtracted from the originally reported RoB values [Herculano-Houzel et al., 2006] the average values obtained here for the olfactory bulb. All values in this study reported for RoB thus correspond to the ensemble of brainstem, diencephalon, and basal ganglia only, and are therefore directly comparable to values provided in our previ- ous studies for primates and insectivores [Herculano-Houzel et al., 2007; Azevedo et al., 2009; Sarko et al., 2009; Gabi et al., 2010]. Similarly, all values for 'whole brain' refer to the sum of cerebral cortex, cerebellum, and RoB, excluding the olfactory bulbs.

\section{Dissection}

All animals were sacrificed by inhalation of ether and perfused transcardially with $0.9 \%$ phosphate-buffered saline followed by $4 \%$ phosphate-buffered paraformaldehyde. Brains were removed from the skull after transecting the spinal cord at the level of the foramen magnum, weighed, and postfixed for 2 weeks to 12 months by immersion in $4 \%$ phosphate-buffered paraformaldehyde. The cerebellum was dissected by cutting the cerebellar peduncles at the surface of the brainstem. Cerebral cortex in all animals was defined as all cortical regions lateral to the olfactory tract, including hippocampus and piriform cortex, and dissected from each hemisphere by peeling it away from the subcortical structures, as described earlier [Herculano-Houzel et al., 2006]. In this manner, the cerebral cortex includes the underlying white matter down to, but not including, the surface of the striatum, therefore excluding the internal capsule. The olfactory bulb was dissected and processed separately (except in the prairie dogs, in which it was not available). All other brain structures (the ensemble of brainstem, diencephalon and striatum) were pooled and processed together as RoB.

\section{Isotropic Fractionator}

Total numbers of cells, neurons, and nonneuronal ('other') cells were estimated as described previously using the isotropic 


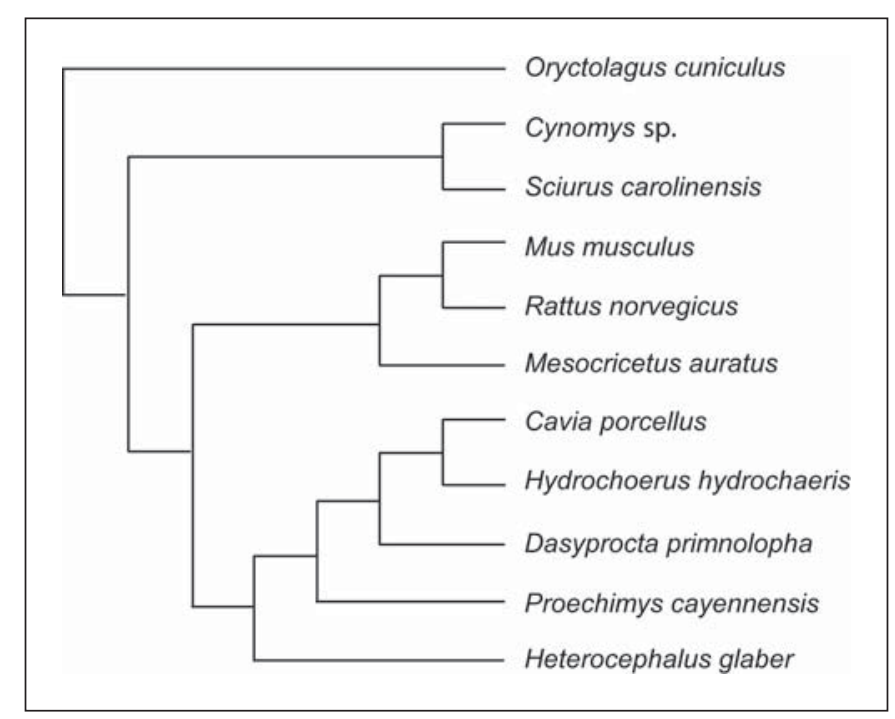

Fig. 1. Phylogenetic relationships between the 11 Glires species examined (data based on Blanga-Kanfi et al. [2009]).

fractionator method [Herculano-Houzel and Lent, 2005]. Briefly, each dissected brain division is turned into an isotropic suspension of isolated nuclei of known, defined volume, kept homogeneous by agitation. The total number of nuclei in suspension - and therefore the total number of cells in the original tissue - is estimated by determining the density of nuclei in small aliquots stained with the fluorescent DNA marker DAPI (4',6-diamidino2-phenylindole dihydrochloride) under the microscope. For each structure, at least four samples of the nuclear suspension are counted independently, in different chambers of a hemocytometer, to determine the number of nuclei/ml of the suspension. The reported values for total number of cells refer to the average nuclei/ml of the samples taken multiplied by the total volume of the suspension. This consistently yields a coefficient of variation of 0.10 , and never more than 0.15 , across samples for a same structure. Once the total cell number is known, the proportion of neurons is determined by immunocytochemical detection of neuronal nuclear antigen (NeuN), expressed in all nuclei of most neuronal cell types and not in nonneuronal cells [Mullen et al., 1992]. Estimates of the proportion of NeuN-positive nuclei are considered reliable since the coefficient of variation among animals of the same species is typically below 0.15 . Numbers of nonneuronal cells are derived by subtraction.

\section{Data Analysis}

All statistical analyses and regressions were performed in Statview (SAS, USA), using the average values obtained for each species. Correlations between variables were calculated using the Spearman correlation coefficient. If a significance criterion of $\mathrm{p}<0.05$ was reached, regressions of the data to power functions were calculated.

\section{Phylogenetic Analysis}

Phylogenetic independent contrasts were calculated to examine the scaling of the brain structures as a function of their cel- lular composition in the expanded dataset of 10 Glires, including the rabbit but excluding the naked mole-rat (see text), while controlling for effects of phylogenetic relatedness in the dataset [Felsenstein, 1985]. Standardized independent contrasts were calculated using the PDAP:PDTREE module of Mesquite software version 2.7 [Maddison and Maddison, 2005]. Contrasts were calculated from log-transformed data to evaluate how well they are described by power functions. Phylogenetic relationships, shown in figure 1, are based on Blanga-Kanfi et al. [2009]. Branch lengths were transformed according to the method of $\mathrm{Pa}$ gel [1992], which assigns all branch lengths to 1 with the constraint that tips are contemporaneous. The reported values for the linear regressions of independent contrasts on log-transformed or raw data are least square regression slope, $\mathrm{r}^{2}$ and $\mathrm{p}$ value. Reduced major axis slopes are similar to the reported least square regression slopes.

\section{Results}

We analyzed the numbers of neurons and other cells that make up the brain of 11 Glires, whose phylogenetic relationships are shown in figure 1 . This dataset expands the original dataset of 6 rodent species [Herculano-Houzel et al., 2006] and includes 10 rodent species belonging to 7 different families (naked mole-rat, Bathyergidae; mouse and rat, Muridae; hamster, Cricetidae; spiny rat, Echimyidae; guinea pig and capybara, Caviidae; prairie dog and grey squirrel, Sciuridae; agouti, Dasyproctidae), and the closely related rabbit (order Lagomorpha). The average data from this expanded dataset of 11 Glires are presented in table 1 divided into four structures: cerebral cortex (grey and white matter combined), cerebellum (grey and white matter combined, including deep nuclei), RoB, and olfactory bulb.

Across the 11 species examined, from the naked mole-rat to the capybara, body mass varies $2,039 \times$, from slightly over $20 \mathrm{~g}$ in the former to almost 50,000 $\mathrm{g}$ in the latter. Brain mass, in turn, is only $191 \times$ larger in the capybara than in the naked mole-rat; cortical mass, $262 \times$; cerebellar mass, $138 \times$, and the RoB, $124 \times$. In contrast, the numbers of neurons in these structures vary disproportionately to brain mass: numbers of neurons are only $58 \times$ larger in the capybara brain than in the naked mole-rat; $50 \times$ larger in the cerebral cortex; $74 \times$ larger in the cerebellum, and $22 \times$ larger in the RoB (table 1). The percentage of neurons in the whole brain decreases significantly with increasing brain size, from a maximum of $65.3 \%$ in the mouse to a minimum of $33.6 \%$ in the capybara (Spearman correlation coefficient: $-0.882, \mathrm{p}=0.0053$ ). 
Conformity to the Cellular Scaling Rules Identified for the Previous Dataset

To examine whether the cellular composition of the brains of the present 4 rodent and 1 Lagomorpha species conform to the rules identified previously, we first recalculated the cellular scaling rules that apply to the original set of 6 rodent species [Herculano-Houzel et al., 2006] by using species averages, instead of the individual values, and now excluding the olfactory bulb from the RoB. These average scaling rules are shown in online supplementary table 1 (for all online suppl. material, see www. karger.com/doi/10.1159/000330825). We find that the exponents recalculated from the species averages are very similar to those reported originally from individual values: $1.748,1.314$ and 1.627 against the original $1.760,1.370$ and 1.772 for the cerebral cortex, cerebellum and RoB, respectively. Because accounting for phylogenetic relatedness in the dataset does not modify the scaling exponents significantly (online suppl. table 2 , previous dataset), with differences of typically only $1-2 \%$, we used the uncorrected scaling functions, obtained directly from the dataset, for further analysis.

Next, we determined how the average numbers of neurons and other cells in each brain structure of the current 5 species depart from the expected values obtained by applying to each species the scaling rules obtained for the previous rodent dataset (power functions shown in online suppl. table 3). For comparison, we also calculated how much the numbers of neurons and other cells depart from the expected values for the brain structures of each of the species in the previous study, given the mass of each structure. Percent deviations from the expected numbers of neurons and other cells were calculated as $[100 \times(\mathrm{ob}-$ served - expected)] for each relationship.

Figure 2 shows that the mass of the cerebral cortex, cerebellum and RoB in the new dataset conform to the expected values from the total brain mass in each species (fig. 2a). Additionally, we find that the spiny rat, the prairie dog, the grey squirrel and the rabbit have numbers of neuronal and other cells that conform to the scaling rules that apply to the previous set of species, with deviations from the expected values that are comparable to those found for the 6 species from which the scaling rules were obtained (fig. 2b, c). Although the squirrel has systematically more neurons and other cells in the cerebral cortex, cerebellum and RoB than expected from the mass of these structures (34.7, 17.5 and $22.4 \%$ more neurons, and $38.2,65.7$ and $86.3 \%$ more other cells, respectively), most of these deviations fall within the range observed for the previous species (fig. 2, sq).

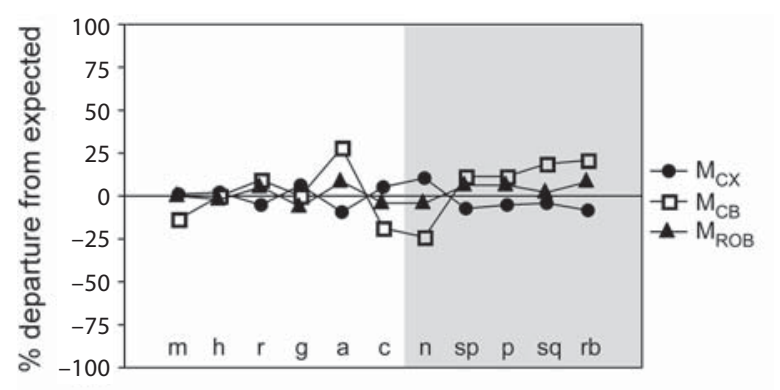

a

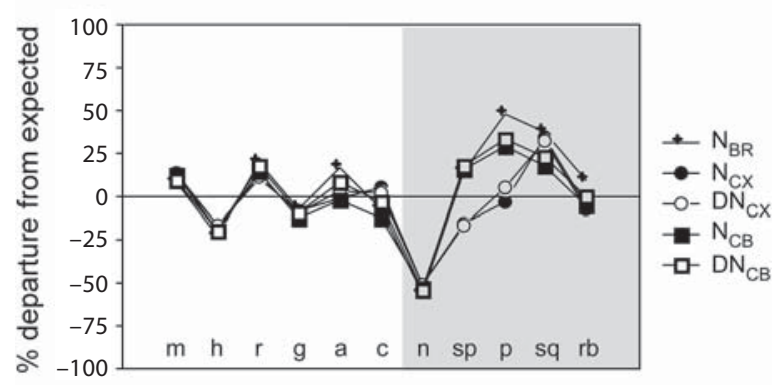

b

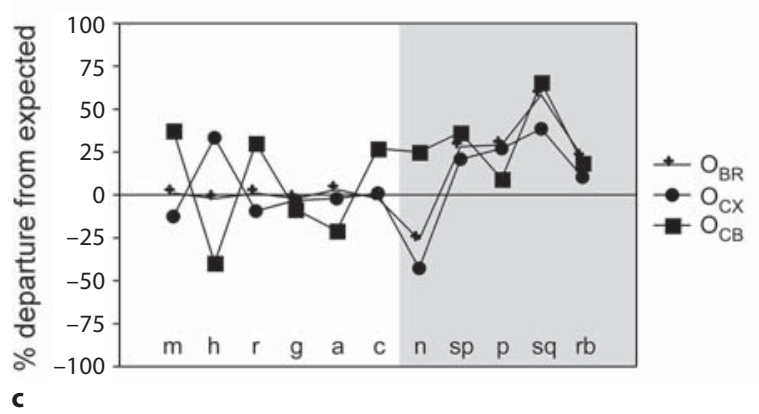

Fig. 2. With the exception of the naked mole-rat, the current Glires deviate from the expected values in their cellular composition by as much as the rodent species studied earlier. The y-axis shows percent deviation from the values expected from the brain scaling rules that apply to the original set of 6 rodent species [HerculanoHouzel et al., 2006]. a Deviation from the expected mass of cerebral cortex $\left(\mathrm{M}_{\mathrm{CX}}\right)$, cerebellum $\left(\mathrm{M}_{\mathrm{CB}}\right)$, and $\mathrm{RoB}\left(\mathrm{M}_{\mathrm{ROB}}\right)$ for a given total brain mass. $\mathbf{b}$ Percent deviation from the expected number of neurons in the brain $\left(\mathrm{N}_{\mathrm{BR}}\right)$, cerebral cortex $\left(\mathrm{N}_{\mathrm{CX}}\right)$ and cerebellum $\left(\mathrm{N}_{\mathrm{CB}}\right)$ as well as in the density of neurons in the cerebral cortex $\left(\mathrm{DN}_{\mathrm{CX}}\right)$ and in the cerebellum $\left(\mathrm{DN}_{\mathrm{CB}}\right)$ calculated from the respective structure mass. $c$ Percent deviation from the expected number of other cells in the brain $\left(\mathrm{O}_{\mathrm{BR}}\right)$, cerebral cortex $\left(\mathrm{O}_{\mathrm{CX}}\right)$ and cerebellum $\left(\mathrm{O}_{\mathrm{CB}}\right)$ calculated from the respective structure mass. Deviations for the 6 rodent species studied earlier are shown in the unshaded area; the 5 new species are shown in the grey area. Species are arranged within each group by order of increasing brain mass. Species key: $\mathrm{m}=$ mouse; $\mathrm{h}$ = hamster; $\mathrm{r}=$ rat; $\mathrm{g}=$ guinea pig; $\mathrm{a}=$ agouti; $\mathrm{c}=$ capybara; $\mathrm{n}=$ naked mole-rat; $\mathrm{sp}=$ spiny rat; $\mathrm{p}=$ prairie dog; $\mathrm{sq}=$ squir$\mathrm{rel} ; \mathrm{rb}=$ rabbit. The power functions used to calculate the expected values are given in the online supplementary table 3. 
Table 2. Updated cellular brain scaling rules calculated from average species values in the expanded Glires dataset

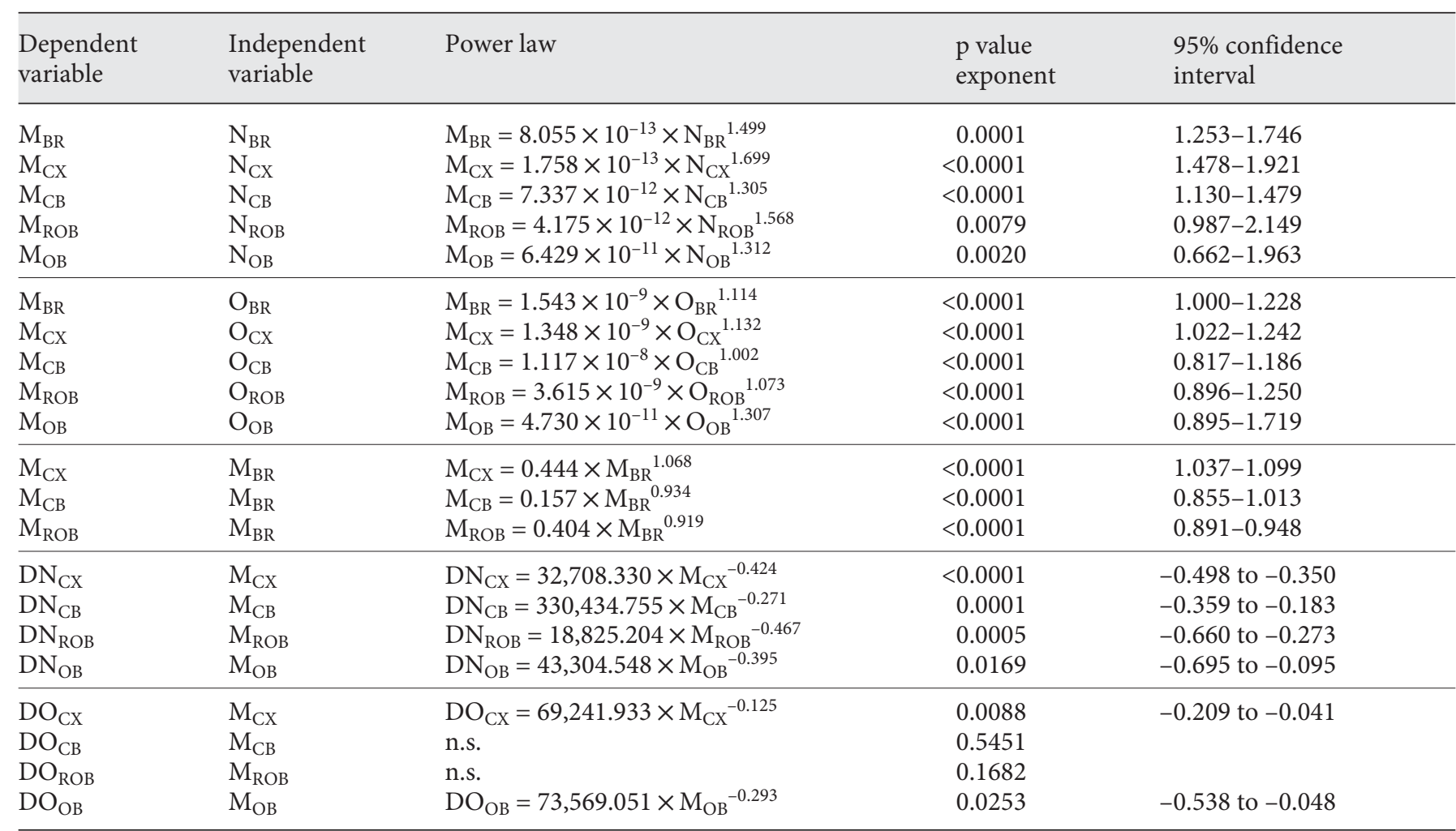

Power laws were calculated from the average species values listed in table 1, originally from Herculano-Houzel et al. [2006]. Species included are mouse, hamster, rat, spiny rat, guinea pig, prairie dog, squirrel, rabbit, agouti and capybara. RoB and whole brain do not include olfactory bulb.

Remarkably, however, we find that the naked mole-rat has about $50 \%$ fewer neurons than expected in the cerebral cortex and cerebellum; this is the largest deviation from the expected values observed for any of the 11 species (fig. 2b, n), with a corresponding deviation of neuronal density in these structures to about $50 \%$ less than expected, given that the mass of the cerebral cortex and cerebellum corresponds to the expected values from total brain mass. In contrast, the number of neurons in the RoB of the naked mole-rat deviates from the expected values for RoB mass by about as much as the species from which these scaling rules were derived $(-32.1 \%$, range: $-48.9 \%$ in hamster to $57.2 \%$ in mouse). These findings indicate that the brains of 4 of the present species, including the closely related lagomorph, conform to the cellular scaling rules described previously for 6 other rodent species, while the naked mole-rat seems to be an outlier, with remarkably fewer neurons than expected in its cerebral cortex and cerebellum.

\section{Updated Cellular Scaling Rules}

Given the conformity of the spiny rat, prairie dog, squirrel and rabbit to the cellular scaling rules identified previously, we next determined the cellular scaling rules that apply to the brains of the expanded dataset of $10 \mathrm{Gli}$ res species (table 2). The naked mole-rat was excluded from the calculations due to its apparent condition as an outlier in the scaling relationships. Importantly, we find that the updated scaling exponents are virtually identical to those identified previously for the smaller set of 6 rodent species (online suppl. table 1), with the expected difference in the neuronal scaling rules that apply to the RoB, which now does not include the olfactory bulb. This similarity to the previous scaling rules further confirms that the 4 species added, including the rabbit, conform to the scaling rules that applied to the previous dataset. This conformity is further seen in the plots of the relationships between structure mass and number of neurons (fig. 3). These plots, which depict the scaling rules calculated 

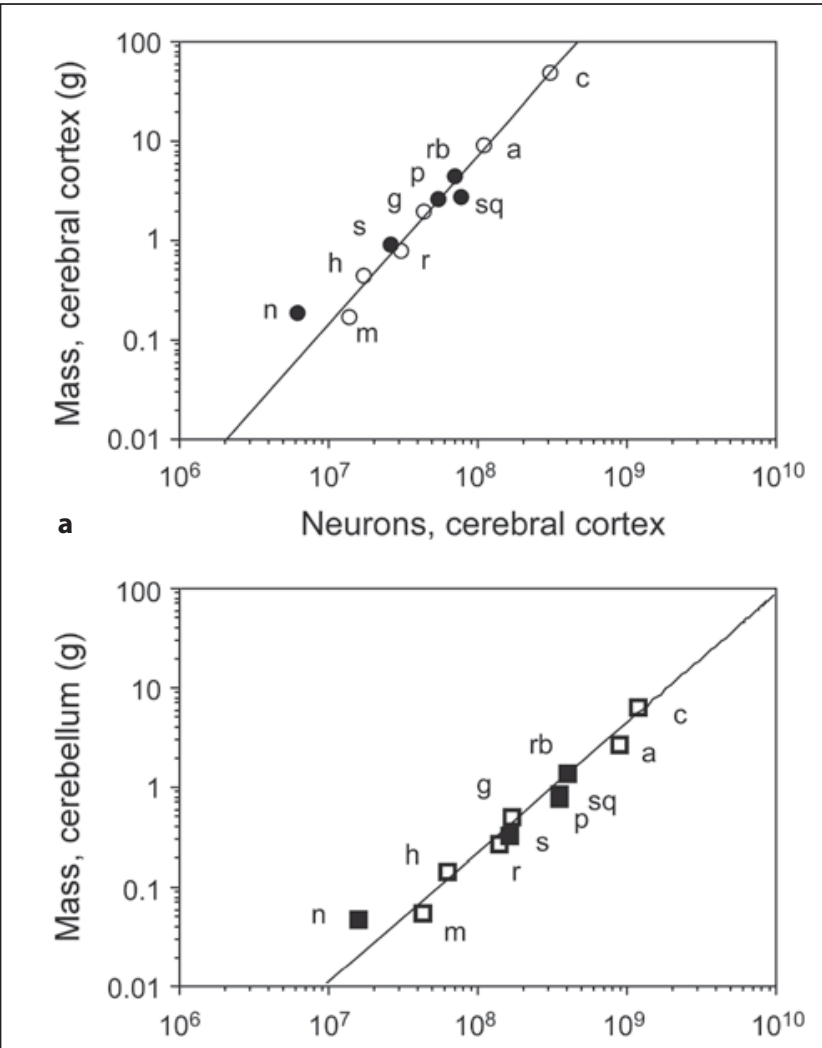

b

Neurons, cerebellum

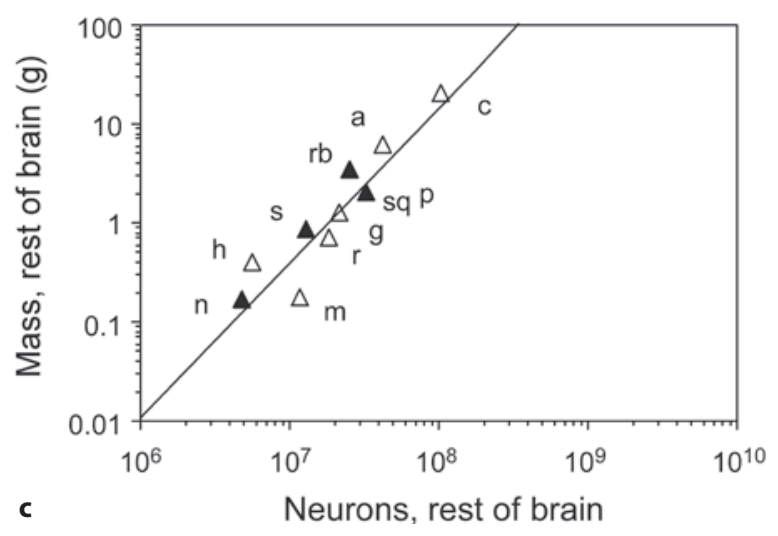

Fig. 3. Scaling of brain structure mass in the combined dataset as a function of numbers of neurons. Each point represents the average mass and number of neurons in the cerebral cortex (a, circles), cerebellum (b, squares) or RoB (c, triangles) of a Glires species. White symbols represent this dataset, black symbols a previous dataset [Herculano-Houzel et al., 2006]. Species key: $\mathrm{m}=$ mouse; $\mathrm{h}=$ hamster; $\mathrm{r}=$ rat; $\mathrm{g}=$ guinea pig; $\mathrm{a}=$ agouti; $\mathrm{c}=$ capybara; $\mathrm{n}=$ naked mole-rat; $\mathrm{sp}=$ spiny rat; $\mathrm{p}=$ prairie dog; $\mathrm{sq}=$ squirrel; $\mathrm{rb}=$ rabbit. Data for the naked mole-rat are shown, but the power functions plotted describe the relationships excluding the naked molerat, as shown in table 2 .

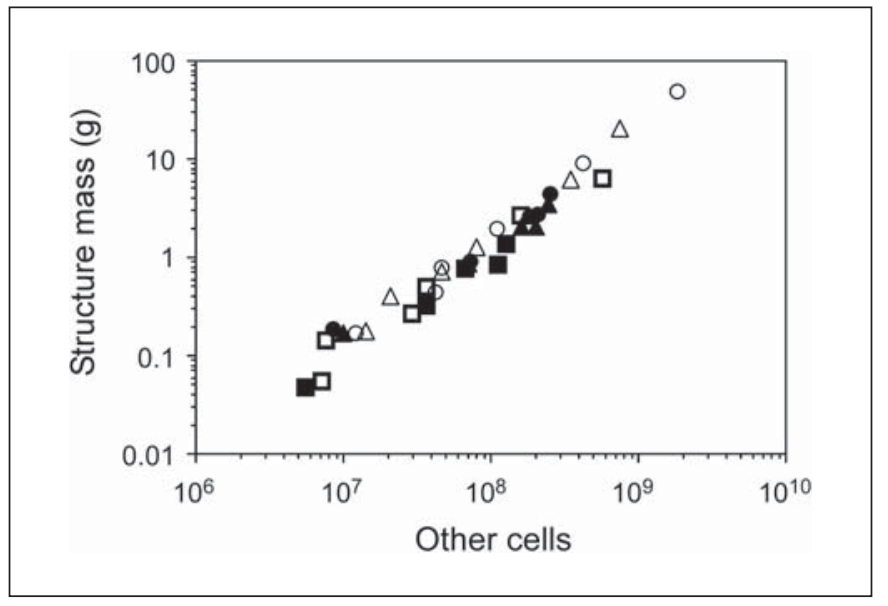

Fig. 4. Scaling of brain structure mass in the combined dataset as a function of numbers of other cells. Each point represents the average mass and number of other cells in the cerebral cortex (circles), cerebellum (squares) or RoB (triangles) of each Glires species. Black symbols represent this dataset, white symbols a previous dataset [Herculano-Houzel et al., 2006]. Power functions are not plotted so as not to obscure the data points, which are largely overlapping across structures.

without the naked mole-rat, also illustrate how the numbers of neurons in the cerebral cortex and cerebellum of this species fall strikingly short of the expected number (fig. 3a, b), while its number of neurons in the RoB is closer to the expected one (fig. 3c). Indeed, adding the naked mole-rat to the analysis alters the neuronal scaling exponents for the cerebral cortex, cerebellum and RoB from $1.699,1.305$ and 1.568 to $1.519,1.160$ and 1.533 , respectively. In contrast to the structure-specific neuronal scaling rules, the functions that relate structure mass to the number of other cells in the structure are remarkably similar to one another (fig. 4). As for the original dataset, these exponents are not significantly affected when phylogenetic relatedness among the species is accounted for (online suppl. table 2).

Separating the olfactory bulb from the RoB allows us to examine the cellular scaling rules that apply to that structure among Glires. We find that the mass of the two olfactory bulbs varies across species as a power function of its number of neurons raised to an exponent of 1.312, and as a power function of its number of other cells raised to an exponent of 1.307 (fig. 5).

In each structure, with the exception of the olfactory bulb, we find that neurons represent decreasing percentages of all cells in the structure with increasing structure mass across species (Spearman correlation coefficients: 


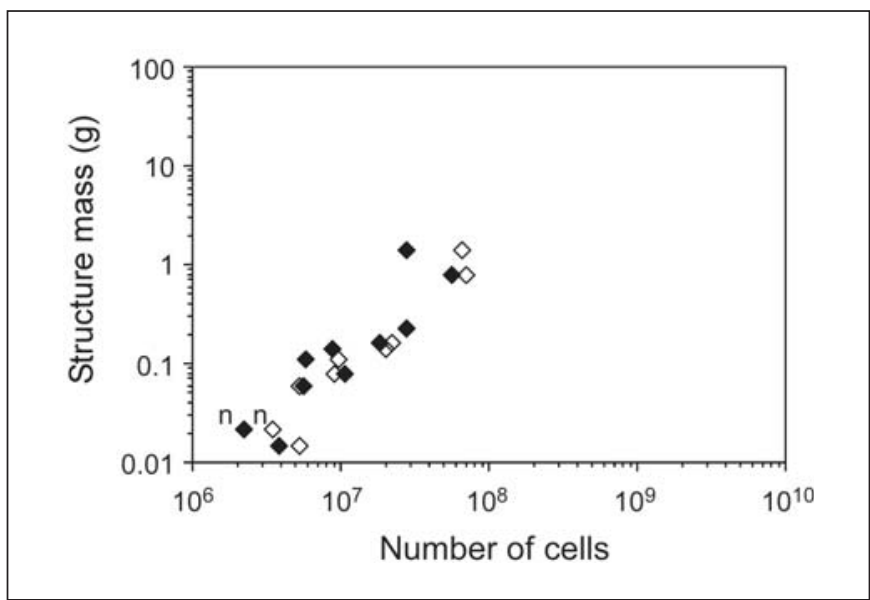

Fig. 5. Scaling of olfactory bulb mass in the combined dataset as a function of numbers of other cells. Each point represents the average mass and number of neurons (black symbols) or other cells (white symbols) in the two olfactory bulbs of each Glires species. Power functions, listed in table 2, are not plotted so as not to obscure the data points. Values for the naked mole-rat are indicated by the letter ' $n$ '.

cerebral cortex, -0.927 ; cerebellum, -0.842 ; RoB, -0.879 , all values of $\mathrm{p}<0.02$; olfactory bulb, $\mathrm{p}=0.2997$ ). Accordingly, the $\mathrm{O} / \mathrm{N}$ ratio (number of other cells/number of neurons) for each structure increases significantly ( $\mathrm{p}<$ 0.05) together with increasing structure mass from a minimum of 0.870 (mouse) to a maximum of 5.983 (capybara) in the cerebral cortex; a minimum of 0.121 (hamster) to a maximum of 0.493 (capybara) in the cerebellum, and a minimum of 1.242 (mouse) to a maximum of 9.638 (rabbit) in the RoB, with no significant correlation with structure mass in the olfactory bulb.

In all structures, neuronal densities decrease with increasing structure mass in nonoverlapping ways that can be described as different power functions of structure mass with negative exponents (table 2; fig. 6a). In contrast, other cell densities are much more overlapping across structures, and do not vary significantly with structure mass (fig. 6b).

\section{Relative Distribution of Brain Mass and Neurons}

Among Glires, the distribution of brain mass changes significantly with increasing brain mass. The relative mass of the cerebral cortex, expressed as the percentage of whole brain mass, increases significantly with increasing brain mass (Spearman correlation coefficient: 0.830, $\mathrm{p}=0.0127$ ), while the relative mass of the RoB decreases

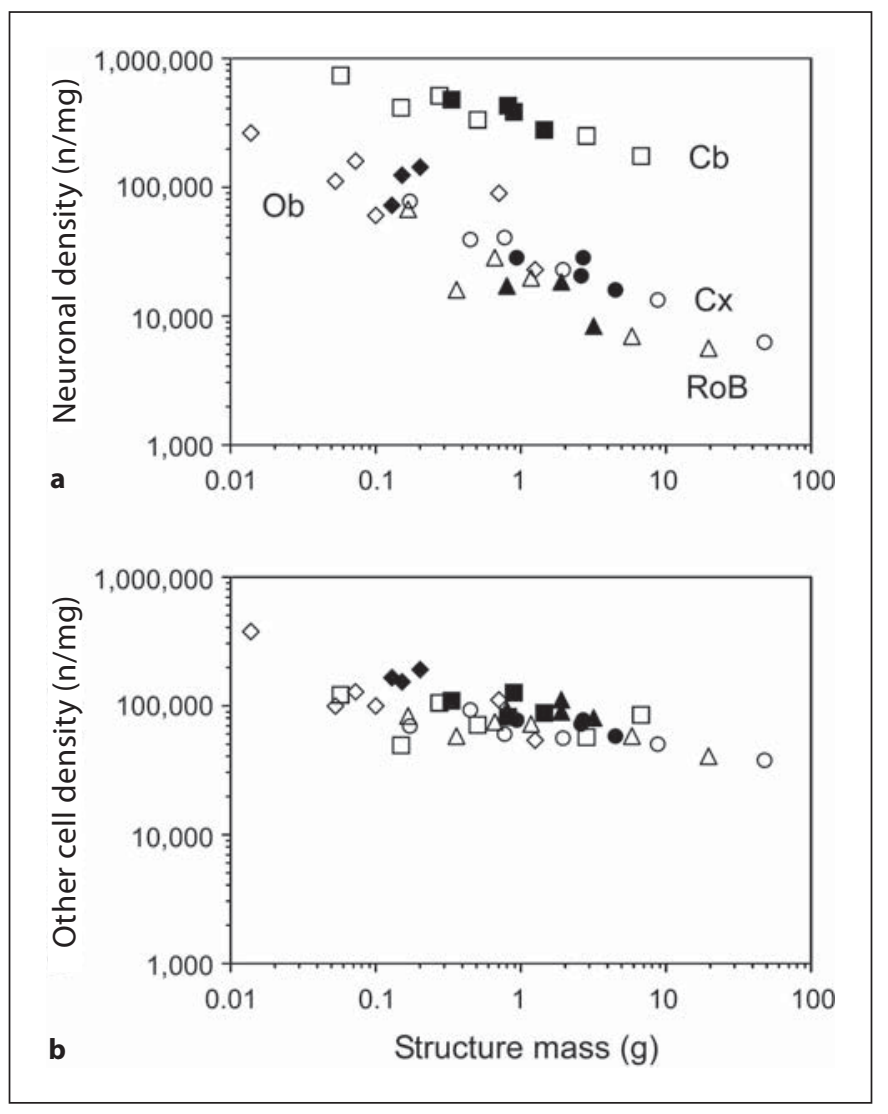

Fig. 6. Neuronal densities are varied and decrease with increasing structure mass, while other cell density is largely overlapping across structures. Each point represents the average mass and neuronal density (a, number of neurons $/ \mathrm{mg}$ ) or other cell density (b, number of other cells/mg) in the cerebral cortex (circles), cerebellum (squares), RoB (triangles) and olfactory bulb (lozenges) in the prior dataset (white symbols) or the current dataset (dark symbols), with the exception of data for the naked mole-rat, which are not plotted. Power functions, listed in table 2, are not plotted so as not to obscure the data points.

significantly (Spearman correlation coefficient: -0.830 , $\mathrm{p}=0.0127)$ and the relative mass of the cerebellum fails to correlate with brain mass (Spearman correlation, $\mathrm{p}=$ 0.8553; naked mole-rat excluded from all calculations). However, the distribution of neurons across these brain structures does not change significantly with increasing brain mass, with no significant correlation found between brain mass and the percent of all brain neurons located in the cerebral cortex $(\mathrm{p}=0.3000)$, cerebellum $(\mathrm{p}=0.1035)$ or $\operatorname{RoB}(\mathrm{p}=0.0710)$. Indeed, relative mass is not correlated with relative number of neurons in the cerebral cortex $(\mathrm{p}=0.9855)$ or in the $\operatorname{RoB}(\mathrm{p}=0.4437)$, and only marginally so in the cerebellum $(\mathrm{p}=0.0325)$. This 
indicates that the relative size is misleading, and the distribution of brain mass is not a valid proxy for the distribution of neurons: relatively larger cerebral cortices in larger Glires brains do not hold relatively more neurons than in smaller brains.

We have recently shown that, despite the faster increase in the mass of the cerebral cortex compared to the cerebellum, numbers of neurons increase coordinately, and linearly, in these two structures across species. We find that, for the expanded dataset of Glires, numbers of neurons also increase coordinately in the two structures in a way that can be described as a power function of the number of neurons in the cerebral cortex with an exponent of 1.101 (which decreases to 1.031 after accounting for phylogenetic relatedness; $p<0.0001$ ), or even better by a linear relationship of slope 3.914 (fig. 7). Remarkably, although the number of neurons in the naked mole-rat cerebral cortex and cerebellum seem smaller than expected from the animal's brain mass (fig. 3), they are still related in the same manner as in other Glires, such that the addition of the naked mole-rat to the dataset leaves the linear slope practically unaltered, at 3.987 (fig. 3).

\section{Brain $\times$ Body Scaling}

After calculating the scaling rules from the average species values, we find that in our previous dataset of 6 rodent species [Herculano-Houzel et al., 2006], brain size increased as a power function of body mass with an exponent of 0.761 ( $\mathrm{p}=0.0006,95 \%$ confidence interval: 0.551-0.971); this exponent is decreased to 0.639 after accounting for phylogenetic relatedness in the dataset $(\mathrm{p}=$ 0.0002 ). For the expanded dataset of 10 species (excluding the naked mole-rat), we find that whole brain mass varies as a power function of body mass with a similar exponent of 0.712 ( $\mathrm{p}<0.0001)$, which also decreases to 0.616 ( $\mathrm{p}<$ 0.0001 ) after accounting for phylogenetic relations. The addition of the naked mole-rat hardly affects the scaling exponents, which become 0.704 before and 0.625 after correcting for phylogenetic relations, respectively $(\mathrm{p}<$ 0.0001). For consistency with the cellular scaling rules for the brain, we shall consider the uncorrected exponent calculated by excluding the naked mole-rat from the dataset.

The number of neurons in the whole brain and the different brain structures of Glires can be predicted from either body mass or brain mass according to the equations described in table 3. Observed numbers of neurons depart from the values predicted from body mass by an average of $30.0 \pm 22.9 \%$, while they depart from the values predicted from brain mass significantly less, by 18.6

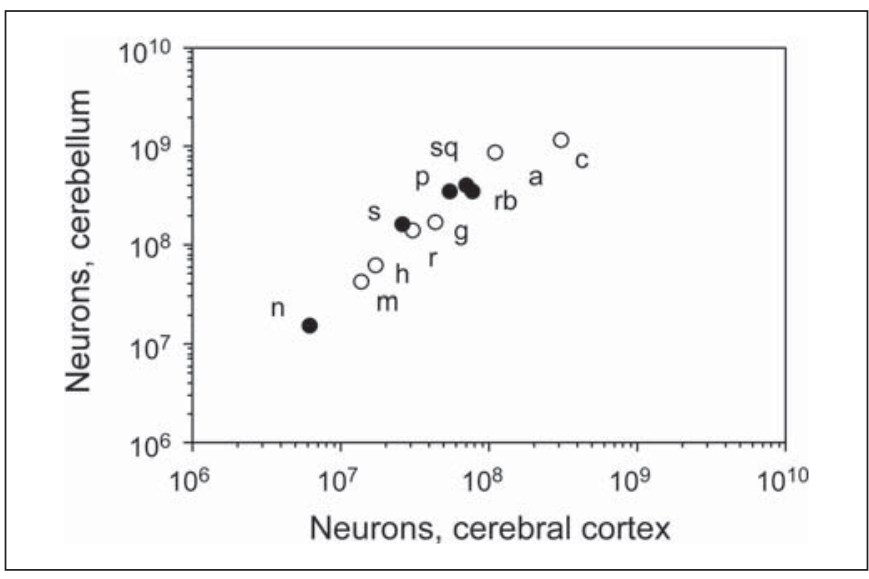

Fig. 7. Coordinated scaling of the numbers of neurons in the cerebellum and cerebral cortex of Glires. Each point represents the average number of neurons in the cerebellum (y-axis) and cerebral cortex (x-axis) of a species (black symbols = current dataset; white symbols = previous dataset). Species key: $\mathrm{m}=$ mouse; $\mathrm{h}=$ hamster; $\mathrm{r}=$ rat; $\mathrm{g}=$ guinea pig; $\mathrm{a}=$ agouti; $\mathrm{c}=$ capybara $\mathrm{n}=$ naked mole-rat; $\mathrm{sp}=$ spiny rat; $\mathrm{p}=$ prairie dog; $\mathrm{sq}=$ squirrel; $\mathrm{rb}=$ rabbit. The scaling is best described as linear, with a slope of 3.914 without the naked mole-rat or 3.987 with it.

$\pm 13.6 \%$ (2-tailed t test, $\mathrm{p}=0.0060)$. Brain mass, therefore, is a better predictor of the number of brain neurons than body mass, which suggests that although brain and body mass are correlated across Glires, body mass is more variable than brain mass for a given number of brain neurons.

\section{Discussion}

Here, we provide a set of updated cellular scaling rules for Glires (rodents and lagomorphs), based on species averages, which, by virtue of excluding the olfactory bulb from the RoB and whole brain, are directly comparable to the cellular scaling rules reported previously for Eulipotyphla and primates [Sarko et al., 2009; Gabi et al., 2010]. Our results demonstrate that, with the notable exception of the naked mole-rat, the 10 rodent species of 6 different families analyzed so far, as well as the closely related rabbit, a lagomorph, conform to the same set of brain scaling rules. Additionally, we find that the scaling exponents that apply to the cellular composition of the brain of Glires are not biased by phylogenetic relatedness in the dataset (for instance, if a subset of the species investigated were closely related and thus skewed the relationship). That the scaling exponents that relate brain 
Table 3. Scaling rules that predict brain neurons from body mass and brain mass calculated from average species values in the expanded Glires dataset

\begin{tabular}{|c|c|c|c|c|}
\hline $\begin{array}{l}\text { Dependent } \\
\text { variable }\end{array}$ & $\begin{array}{l}\text { Independent } \\
\text { variable }\end{array}$ & Power law & $\begin{array}{l}\mathrm{p} \text { value } \\
\text { exponent }\end{array}$ & $\begin{array}{l}95 \% \text { confidence } \\
\text { interval }\end{array}$ \\
\hline $\mathrm{M}_{\mathrm{BR}}$ & $\mathrm{M}_{\mathrm{BD}}$ & $\mathrm{M}_{\mathrm{BR}}=0.037 \times \mathrm{M}_{\mathrm{BD}}^{0.712}$ & $<0.0001$ & $0.549-0.875$ \\
\hline $\mathrm{M}_{\mathrm{CB}}$ & $\mathrm{M}_{\mathrm{BD}}$ & $\mathrm{M}_{\mathrm{CB}}=0.008 \times \mathrm{M}_{\mathrm{BD}}^{0.660}$ & $<0.0001$ & $0.483-0.836$ \\
\hline $\mathrm{M}_{\mathrm{ROB}}$ & $\mathrm{M}_{\mathrm{BD}}$ & $\mathrm{M}_{\mathrm{ROB}}=0.020 \times \mathrm{M}_{\mathrm{BD}}{ }^{0.656}$ & $<0.0001$ & $0.508-0.803$ \\
\hline $\mathrm{N}_{\mathrm{CB}}$ & $\mathrm{M}_{\mathrm{BD}}$ & $\mathrm{N}_{\mathrm{CB}}=9,517,291.663 \times \mathrm{M}_{\mathrm{BD}}{ }^{0.482}$ & 0.0002 & $0.310-0.653$ \\
\hline $\mathrm{N}_{\mathrm{ROB}}$ & $\mathrm{M}_{\mathrm{BD}}$ & $\mathrm{N}_{\mathrm{ROB}}=2,495,688.704 \times \mathrm{M}_{\mathrm{BD}} 0.339$ & 0.0015 & $0.173-0.504$ \\
\hline $\mathrm{N}_{\mathrm{BR}}$ & $\mathrm{M}_{\mathrm{BR}}$ & $\mathrm{N}_{\mathrm{BR}}=120,800,578.409 \times \mathrm{M}_{\mathrm{BR}}^{0.641}$ & $<0.0001$ & $0.535-0.746$ \\
\hline $\mathrm{N}_{\mathrm{CX}}$ & $\mathrm{M}_{\mathrm{BR}}$ & $\mathrm{N}_{\mathrm{CX}}=20,352,603.956 \times \mathrm{M}_{\mathrm{BR}}^{0.613}$ & $<0.0001$ & $0.531-0.694$ \\
\hline $\mathrm{N}_{\mathrm{CB}}$ & $\mathrm{M}_{\mathrm{BR}}$ & $\mathrm{N}_{\mathrm{CB}}=86,121,138.094 \times \mathrm{M}_{\mathrm{BR}}^{0.691}$ & $<0.0001$ & $0.557-0.825$ \\
\hline
\end{tabular}

Power laws were calculated from the average species values listed in table 1, originally from Herculano-Houzel et al. [2006]. Species included are mouse, hamster, rat, spiny rat, guinea pig, prairie dog, squirrel, rabbit, agouti and capybara. RoB and whole brain do not include olfactory bulb.

mass to body mass are more affected than the cellular scaling rules by accounting for phylogenetic relatedness in our dataset suggests that the cellular scaling rules uncovered here apply to Glires in a more general manner than the brain $x$ body mass relationships among species.

A comparison of the cellular scaling rules observed for primates, insectivores and Glires shows that the cerebral cortex varies in mass as different power functions of its number of neurons in the different orders, with exponents of 1.0, 1.6 and 1.7, respectively [Sarko et al., 2009; Gabi et al., 2010]. Cerebellar mass, in turn, scales in the different orders with its number of neurons raised to exponents of 1.0, 1.0 and 1.3. Although different exponents are also found to relate the mass of the RoB to its number of neurons across the three orders $(1.4,1.0$ and 1.6 in primates, insectivores and Glires, respectively), the equations that describe this relationship are less clearly discernible across the three orders than the neuronal scaling rules that apply to the cerebral cortex and cerebellum. This raises the interesting possibility that the ensemble of brainstem, diencephalon and striatum, the evolutionarily oldest structures in the brain, share conserved neuronal scaling rules across the three mammalian orders; this is possibly related to conserved functions of these structures across mammals, while the more recent cerebral cortex and cerebellum have evolved different scaling rules in each order, possibly related, for instance, to environmental requirements and selective pressure that are particular to each group. Importantly, we confirm our previous observation that the relative expansion of the cerebral cortex in larger brains does not reflect a relative expansion in the number of cortical neurons, and find that the linear relationship between numbers of cerebellar and cerebral cortical neurons observed in the previous set of 6 rodent species also applies to the current extended dataset of Glires, with the same average of about 4 cerebellar neurons to every neuron in the cerebral cortex [Herculano-Houzel, 2010]. This coordinate increase in numbers of neurons in the cerebral cortex and cerebellum adds support to the notion that the two structures operate together, and have also been evolving together [Whiting and Barton, 2003; Ramnani et al., 2006; Balsters et al., 2010].

\section{The Outlier: Naked Mole-Rat}

The conserved scaling relationships between brain size and numbers of neurons among Glires, primates or insectivores are presumably due to genetic factors that regulate development within and across mammalian orders [Riska and Atchley, 1985; Bi et al., 2009; Gaglani et al., 2009]. We do not assume that life history and mode of living have no effect on the cellular composition of the brain or brain size; in fact, several environmental factors are know to impact the size of brain structures [e.g. Maguire et al., 2000; Campi and Krubitzer, 2010; AbdulKareem et al., 2011]. In most cases, however, these effects 
are likely to be rather insignificant compared to the over 1,000 -fold variation we investigate. It is remarkable, therefore, that here we encounter for the first time a clear outlier in the cellular scaling relationships that apply to mammalian species: the African naked mole-rat, which, despite having the expected distribution of mass among its brain regions (that is, a normally proportioned cerebral cortex, cerebellum and RoB), contains only half of the number of neurons expected in the cerebral cortex and cerebellum from the mass of these structures. Although such a discrepancy could be accounted for if a different set of neuronal scaling rules applied to the cerebral cortex and cerebellum of this species than to other Glires, a more likely explanation is that the discrepancy is due to regressive events, related to the strictly fossorial lifestyle of this species, which would presumably occur after the development of a normal complement of brain neurons according to the same scaling rules that apply to Glires as a whole. The naked mole-rat has reduced eyes, a lateral geniculate nucleus that is reduced in volume by about a third due to regressive changes [Xiao et al., 2006], and a reduced visual cortex that is apparently taken over by a relatively enlarged primary somatosensory cortex [Catania and Remple, 2002], or possibly lost. Thus, the $50 \%$ smaller number of cortical neurons relative to the expected number in the naked mole-rat might be related to the regression of the retinogeniculocortical system compared to the other rodents that rely heavily on their visual system [Xiao et al., 2006], possibly leading to neuronal loss in the cerebral cortex. Interestingly, the similar 50\% reduction in the number of neurons in the naked mole-rat cerebellum is compatible with our previous suggestion that the number of neurons in this structure might be adjusted proportionately to the number of cerebral cortical neurons during the development of each individual [Herculano-Houzel, 2010]. However, regressive events in the cerebellum directly related to selective pressure, given the fossorial lifestyle of this species, cannot at the moment be ruled out. Additionally, the decreased numbers of cortical neurons in the naked mole-rat brain relative to the expected numbers may also be related to the metabolic costs of life under chronic environmental hypoxia due to burrowing, even if globin expression in the brain is changed as an adaptation that makes hypoxia more tolerable [Avivi et al., 2010].

\section{Predictions for Extinct Giant Rodent Species}

The Amazonian capybara is currently the largest living rodent, but it is by no means the largest rodent ever: three now extinct larger rodent species that once roamed the Americas are the Castoroides, estimated to weigh up to $200 \mathrm{~kg}$ [Reynolds, 2002], Phoberomys, estimated to weigh $700 \mathrm{~kg}$ [Sánchez-Villagra et al., 2003], and Josephoartigasia, predicted toweigh about $1,200 \mathrm{~kg}$ [Rinderknecht and Blanco, 2008]. The estimated time of divergence of these species from their common ancestor with the species in the present dataset implies that the same cellular scaling rules that apply to the brains of modern Glires must also have applied to them. This allows one to use the present scaling rules to predict the neuronal composition of the brain of these species from their body size. Keeping in mind that these predictions match observed values with an average error of $30 \%$ for the extant Glires, it can be estimated from their calculated body masses that the giant Castoroides, Phoberomys, and Josephoartigasia had brains weighing 220, 537 and 793 g, respectively, holding a total of 3.7, 6.6 and 8.4 billion neurons. For the sake of comparison, we have recently estimated that the gorilla brain, at $486 \mathrm{~g}$, holds approximately 33 billion neurons [Herculano-Houzel and Kaas, 2011]. Indeed, hypothetical primates with similar brain weights of 200,537 and $793 \mathrm{~g}$ would be expected to contain between 4 and 9 times more neurons: 16, 46 and 76 billion, respectively [based on Herculano-Houzel et al., 2007]. Compared to the cellular scaling rules that apply to primates, therefore, the rodent/lagomorph way of building large brains is found to be very volume-expensive, with larger numbers of neurons coming at a great volumetric cost (or, alternatively, with greater brains offering a disproportionately small benefit of added neurons). Without a corresponding benefit of proportionately larger numbers of brain neurons, such an inflationary scaling of brain size relative to its number of neurons, especially when associated with a very large body that had to be metabolically sustained, may have imposed serious constraints on giant Glires that may have contributed to their extinction.

\section{Acknowledgements}

We thank Prof. Mohammad Z. Abedin for providing the prairie dog brains, and Ingrid Croaro for providing the Proechimys brains. This work was supported by FAPERJ/Cientista do Nosso Estado, $\mathrm{CNPq}$ - Edital Universal and Bolsa Produtividade, Instituto Nacional de Ciência e Tecnologia (MCT/CNPq/FAPESP) and the James S. McDonnell Foundation (S.H.H.), NSF grant 0844743 (K.C.) and NEI grant EY02686 (J.H.K.). 


\section{References}

-Abdul-Kareem IA, Stancak A, Parkes LM, Sluming V (2011): Increased gray matter volume of pars opercularis in male orchestral musicians correlate positively with years of musical performance. J Magn Reson Imaging 33: $24-32$.

-Avivi A, Gerlach F, Joel A, Reuss S, Burmester T, Nevo E, Hankeln T (2010): Neuroglobin, cytoglobin, and myoglobin contribute to hypoxia adaptation of the subterranean mole rat Spalax. Proc Natl Acad Sci USA 107: 21570-21575.

-Azevedo FA, Carvalho LR, Grinberg LT, Farfel JM, Ferretti RE, Leite RE, Jacob Filho W, Lent R, Herculano-Houzel S (2009): Equal numbers of neuronal and nonneuronal cells make the human brain an isometrically scaled-up primate brain. J Comp Neurol 513: 532-541.

- Balsters JH, Cussans E, Diedrichsen J, Phillips KA, Preuss TM, Rilling JK, Ramnani N (2010): Evolution of the cerebellar cortex: the selective expansion of prefrontal-projecting cerebellar lobules. Neuroimage 49:20452052.

-Bi W, Sapir T, Shchelochkov OA, Zhang F, Withers MA, Hunter JV, Levy T, Shinder V, Peiffer DA, Gunderson KL, Nezarati MM, Shotts VA, Amato SS, Savage SK, Harris DJ, DaySalvatore DL, Horner M, Lu XY, Sahoo T, Yanagawa Y, Beaudet AL, Cheung SW, Martinez S, Lupski JR, Reiner O (2009): Increased LIS1 expression affects human and mouse brain development. Nat Genet 2:168177.

-Blanga-Kanfi S, Miranda H, Penn O, Pupko T, DeBry RW, Huchon D (2009): Rodent phylogeny revised: analysis of six nuclear genes from all major rodent clades. BMC Evol Biol 9:71.

-Campi KL, Krubitzer L (2010): Comparative studies of diurnal and nocturnal rodents: differences in lifestyle result in alterations in cortical field size and number. J Comp Neurol 518:4491-4512.

-Catania KC, Remple MS (2002): Somatosensory cortex dominated by the representation of teeth in the naked mole-rat brain. Proc Natl Acad Sci USA 99:5692-5697.

Clark DA, Mitra PP, Wang SS (2001): Scalable architecture in mammalian brains. Nature 411:189-193.

Douzery EJP, Delsuc F, Stanhope MJ, Huchon D (2003): Local molecular clocks in three nuclear genes: divergence times for rodents and other mammals and incompatibility among fossil calibrations. J Mol Evol 57:S201.

Douzery EJP, Huchon D (2004): Rabbits, if anything, are likely Glires. Mol Phylogenet Evol 33:922-935.

Felsenstein J (1985): Phylogenies and the comparative method. Am Nat 125:1-15.
Fox JH, Wilczynski W (1986): Allometry of major CNS divisions: towards a reevaluation of somatic brain-body scaling. Brain Behav Evol 28:157-169.

Gabi M, Collins CE, Wong P, Torres LB, Kaas JH, Herculano-Houzel S (2010): Cellular scaling rules for the brains of an extended number of primate species. Brain Behav Evol 76:32-44.

Gaglani SM, Lu L, Williams RW, Rosen GD (2009): The genetic control of neocortex volume and covariation with neocortical gene expression in mice. BMC Neurosci 10:44.

Haug H (1987): Brain sizes, surfaces, and neuronal sizes of the cortex cerebri: a stereological investigation of man and his variability and a comparison with some mammals (primates, whales, marsupials, insectivores, and one elephant). Am J Anat 180:126-142.

Herculano-Houzel S (2010): Coordinated scaling of cortical and cerebellar numbers of neurons. Front Neuroanat 4:12.

-Herculano-Houzel S, Collins CE, Wong P, Kaas JH (2007): Cellular scaling rules for primate brains. Proc Natl Acad Sci USA 104:35623567.

Herculano-Houzel S, Kaas JH (2011): Gorilla and orangutan brains conform to the primate cellular scaling rules: implications for human evolution. Brain Behav Evol 77:3344.

-Herculano-Houzel S, Lent R (2005): Isotropic fractionator: a simple, rapid method for the quantification of total cell and neuron numbers in the brain. J Neurosci 25:2518-2521.

Herculano-Houzel S, Mota B, Lent R (2006): Cellular scaling rules for rodent brains. Proc Natl Acad Sci USA 103:12138-12143.

-Horner DS, Lefkimmiatis K, Reyes A, Gissi C, Saccone C, Pesole G (2007): Phylogenetic analyses of complete mitochondrial genome sequences suggest a basal divergence of the enigmatic rodent Anomalurus. BMC Evol Biol 7:16.

Kruska DCT (2007): The effects of domestication on brain size; in Krubitzer L, Kaas J (eds): Evolution of Nervous Systems, vol 3, pp 143-153.

Kullberg M, Nilsson MA, Arnason U, Harley EH, Janke A (2006): Housekeeping genes for phylogenetic analysis of eutherian relationships. Mol Biol Evol 23:1493-1503.

Lin Y-H, McLenachan PA, Gore AR, Phillips MJ, Ota R, Hendy MD, Penny D (2002): Four new mitochondrial genomes and the increased stability of evolutionary trees of mammals from improved taxon sampling. Mol Biol Evol 19:2060-2070.

Liu FG, Miyamoto MM (1999): Phylogenetic assessment of molecular and morphological data for eutherian mammals. Syst Biol 48: 54-64.

Maddison WP, Maddison DR (2005): Mesquite: a modular system for evolutionary analysis. Version 2.7. http://mesquite project.org.
Maguire EA, Gadian DG, Johnsrude IS, Good CD, Ashburner J, Frackowiak RS, Frith CD (2000): Navigation-related structural change in the hippocampi of taxi drivers. Proc Natl Acad Sci USA 97:4398-4403.

Marino L (2006): Absolute brain size: did we throw the baby out with the bathwater? Proc Natl Acad Sci USA 103:13563-13564.

Martin RD (1981): Relative brain size and basal metabolic rate in terrestrial vertebrates. $\mathrm{Na}$ ture 293:57-60.

Mullen RJ, Buck CR, Smith AM (1992): NeuN, a neuronal specific nuclear protein in vertebrates. Development 116:201-211.

Pagel MD (1992): A method for the analysis of comparative data. J Theor Biol 156:431-442.

-Ramnani N, Behrens TE, Johansen-Berg H, Richter MC, Pinsk MA, Andersson JL, Rudebeck P, Ciccarelli O, Richter W, Thompson AJ, Gross CG, Robson MD, Kastner S, Matthews PM (2006): The evolution of prefrontal inputs to the cortico-pontine system: diffusion imaging evidence from macaque monkeys and humans. Cereb Cortex 16:811-818.

Reynolds PS (2002): How big is a giant? The importance of method in estimating body size of extinct mammals. J Mammal 83:321-332.

Rinderknecht A, Blanco RE (2008): The largest fossil rodent. Proc R Soc B 275:923-928.

Riska B, Atchley WR (1985): Genetics of growth predict patterns of brain-size evolution. Science 229:668-671.

Sánchez-Villagra MR, Aguilera O, Horovitz I (2003): The anatomy of the world's largest extinct rodent. Science 301:1708-1710.

-Sarko DK, Catania KC, Leitch DB, Kaas JH, Herculano-Houzel S (2009): Cellular scaling rules of insectivore brains. Front Neuroanat 3:8.

Stephan H, Frahm H, Baron G (1981): New and revised data on volumes of brain structures in insectivores and primates. Folia Primatol 35:1-29.

-Stolzenburg JU, Reichenbach A, Neumann M (1989): Size and density of glial and neuronal cells within the cerebral neocortex of various insectivorian species. Glia 2:78-84

Tower DB, Elliot KAC (1952): Activity of acetylcholine system in cerebral cortex of various unanesthetized mammals. Am J Physiol 168 747-759.

Whiting BA, Barton RA (2003): The evolution of the cortico-cerebellar complex in primates: anatomical connections predict patterns of correlated evolution. J Hum Evol 44:3-10.

Wilson DE, Reeder DM (2005): Mammal Species of the World: A Taxonomic and Geographic Reference. Baltimore, John Hopkins University Press.

Xiao J, Levitt JB, Buffenstein R (2006): The use of a novel and simple method of revealing neural fibers to show the regression of the lateral geniculate nucleus in the naked molerat (Heterocephalus glaber). Brain Res 1077: 81-89. 